\title{
Dynamic Magnetization Switching in NiO Nanoparticles: Pulsed Field Magnetometry Study
}

D. A. Balaev ${ }^{1,2}$, A. A. Krasikov¹, A. A. Dubrovskii ${ }^{1,2}$, A. D. Balaev ${ }^{1}$, S. I. Popkov ${ }^{1,2}$, V. L. Kirillov ${ }^{3}$, and O. N. Martyanov ${ }^{3}$

${ }^{1}$ Kirensky Institute of Physics, Federal Research Center KSC SB RAS, Krasnoyarsk, 660036 Russia

${ }^{2}$ Siberian Federal University, Krasnoyarsk, 660041 Russia

${ }^{3}$ Boreskov Institute of Catalysis, Russian Academy of Sciences, Siberian Branch, Novosibirsk, 630090 Russia

e-mail: dabalaev@iph.krasn.ru

\begin{abstract}
The dynamic magnetization switching of antiferromagnetic nickel oxide nanoparticles with a characteristic size of $8 \mathrm{~nm}$ has been experimentally investigated by pulsed field magnetometry. It is shown that, due to the presence of defects in $\mathrm{NiO}$ nanoparticles, as in other antiferromagnetic particles, the uncompensated magnetic moment is induced by the incomplete compensation of spins at the antiferromagnetic ordering. The dynamic magnetic hysteresis loops have been studied in pulsed fields with the maximum field $\mathrm{H}_{\max }$ of up to $130 \mathrm{kOe}$ and pulse lengths $\tau_{\mathrm{P}}$ of 4 , 8, and $16 \mathrm{~ms}$. According to the results obtained, the coercivity $H_{C}$ depends on both the $\tau_{P}$ and $H_{\max }$ values. The observed increase in the $\mathrm{H}_{\mathrm{C}}$ value with decreasing pulse length (i.e., with increasing switching field frequency) is unambiguously related with the relaxation processes typical of single-domain ferromagnetic nanoparticles. However, the observed effect of the maximum applied field $\mathrm{H}_{\max }$ on the $\mathrm{H}_{\mathrm{C}}$ value is assumed to be a feature of antiferromagnetic nanoparticles.
\end{abstract}

\section{Introduction}

It is well-known that the physical properties of nanomaterials can drastically differ from the properties of their bulk analogs. This is valid, in particular, for magnetic nanoparticles. A spectacular example of objects with different types of magnetic ordering is nanoparticles of antiferromagnetically (AFM) ordered materials [1,2]. The main difference of AFM nanoparticles from bulk analogs is their uncompensated magnetic moment, which can result, on the one hand, from the developed surface with uncompensated chemical bonds and different local environment of atoms on the particle surface and, on the other hand, from the existence of defects both on the particle surface and in the bulk of particles. All the aforementioned violates the compensation of spin magnetic moments of sublattices typical of the AFM ordering; as a result, the particle acquires uncompensated magnetic moment $\mu_{\mathrm{p}}$, which can attain several hundreds of Bohr magnetons at a particle size of a few nanometers. Thus, small AFM particles become magnetic, which opens wide opportunities for application of these materials $[3,4]$.

The magnetic properties of AFM nanoparticles are similar to the properties of ferro- and ferrimagnetic nanoparticles [1]. The magnetic moments of AFM particles can be in the so-called unblocked (superparamagnetic (SP)) and blocked states. Critical volume V and blocking temperature $\mathrm{T}_{\mathrm{B}}$ of these states are related to the characteristic particle relaxation time $\tau_{0}\left(\tau_{0} \sim 10^{-9 \div 12} \mathrm{~s}\right.$ [1]), magnetic anisotropy constant $\mathrm{K}$, and time $\tau_{\mathrm{m}}$ depending on a magnetic measurement technique used by the Nèel-Brown formula

$$
\mathrm{T}_{\mathrm{B}}=\mathrm{KV} / \ln \left(\tau_{\mathrm{m}} / \tau_{0}\right) k_{\mathrm{B}},
$$

where $k_{\mathrm{B}}$ is the Boltzmann constant. In the temperature range of $\mathrm{T}<\mathrm{T}_{\mathrm{B}}$, the magnetization hysteresis $\mathrm{M}(\mathrm{H})$ is observed. In the temperature range of $\mathrm{T}>\mathrm{T}_{\mathrm{B}}$, the $\mathrm{M}(\mathrm{H})$ dependences are reversible and magnetization curves can be described by the classical Langevin function. In the case of AFM nanoparticles, the $\mathrm{M}(\mathrm{H})$ dependences should be described taking into account the linear-in-field contribution of the AFM core [1,2,5-12]. 
In the techniques for studying the magnetic properties, including magnetometry, ac susceptibility, Mössbauer effect, and magnetic resonance, the characteristic $\tau_{\mathrm{m}}$ values in Eq. (1) are different. The possibility of changing this parameter can yield new information, since it concerns the relaxation processes [13-15]. In standard quasi-steady-state magnetic measurements, we have $\tau_{\mathrm{m}}$ $\sim 10^{1-2} \mathrm{~s}[1]$. In the ac susceptibility technique, $\tau_{\mathrm{m}}$ is determined by ac field frequency $\omega: \tau_{\mathrm{m}}=2 \pi / \omega$. The magnetic hysteresis loop can also be measured in ac fields; in this case, however, a significant limitation is imposed to the maximum field, which is usually no stronger than $\sim 10^{3}$ Oe. Using the pulsed technique, one can obtain much stronger (by, at least, two orders of magnitude) magnetic fields and thereby get more information about the magnetic behavior.

In our previous work [16], we studied the dynamic magnetization switching of $\varepsilon-\mathrm{Fe}_{2} \mathrm{O}_{3}$ nanoparticles in pulsed magnetic fields using the similar approach [16]. The dependences of the coercivity on the external field variation rate $\mathrm{dH} / \mathrm{dt}$ were described in the framework of a theoretical model of dynamic switching of ferromagnetic nanoparticles [13, 14]. The data obtained [16] unambiguously revealed a key role of surface effects in the formation of dynamic properties of the magnetic oxide nanoparticles [17, 18]. In addition, we found a certain discrepancy between the theoretical model and the observed characteristics of a system under temperature drop to $77 \mathrm{~K}$. In this study, we extend these experimental approaches to AFM NiO particles with a characteristic size of about $8 \mathrm{~nm}$. The aim of this study was to establish the regularities of the dynamic magnetization switching of the AFM oxide nanoparticles by comparing the data obtained with the results of other examination methods.

\section{Experimental}

\subsection{Preparing and characterizing nanosized $\mathrm{NiO}$}

The $\mathrm{NiO}$ nanoparticles were formed by thermal decomposition of nickel oxalate $\mathrm{NiC}_{2} \mathrm{O}_{4} \cdot 2 \mathrm{H}_{2} \mathrm{O}$ obtained from $\mathrm{NiSO}_{4} \cdot 7 \mathrm{H}_{2} \mathrm{O}(\mathrm{P})$ and $\left(\mathrm{NH}_{4}\right)_{2} \mathrm{C}_{2} \mathrm{O}_{4} \cdot \mathrm{H}_{2} \mathrm{O}(\mathrm{AR})$ taken in the stoichiometric ratio. To prepare the desired mixture, the $14-\% \quad \mathrm{NiSO}_{4} \cdot 7 \mathrm{H}_{2} \mathrm{O}$ solution was added with the corresponding amount $(3.6 \%)$ of the $\left(\mathrm{NH}_{4}\right)_{2} \mathrm{C}_{2} \mathrm{O}_{4} \cdot \mathrm{H}_{2} \mathrm{O}$ solution upon mixing at a rate of $250 \mathrm{rpm}$ for $20 \mathrm{~min}$. The obtained light-green precipitate was filtered through a paper filter with a pore diameter of 1-2.5 $\mu \mathrm{m}$ and washed from $\left(\mathrm{NH}_{4}\right)_{2} \mathrm{SO}_{4}$ with distilled water. The decomposition conditions were the temperature growth to $400^{\circ} \mathrm{C}$ for $40 \mathrm{~min}$ and 10 -min exposure at this temperature.

The X-ray diffraction (XRD) pattern for the NiO nanoparticle sample was obtained on a Bruker D8 Advance (Germany) diffractometer $(\mathrm{CuK} \alpha$ radiation, $\lambda=1.5418 \AA$ (Fig. 1). All the diffraction peaks correspond to the $\mathrm{NiO}$ phase (PDF no. 047-1049). The $\mathrm{NiO}$ cubic unit cell parameter is consistent with a reference value (sp. gr. Fm $\overline{3} \mathrm{~m}, \mathrm{a}=\mathrm{b}=\mathrm{c}=4.176 \AA, \alpha=\beta=\gamma=90^{\circ}$ ). The mean size of the coherent scattering region of crystallites calculated from the XRD peak halfwidths using the Scherrer equation was around $8 \mathrm{~nm}$. 


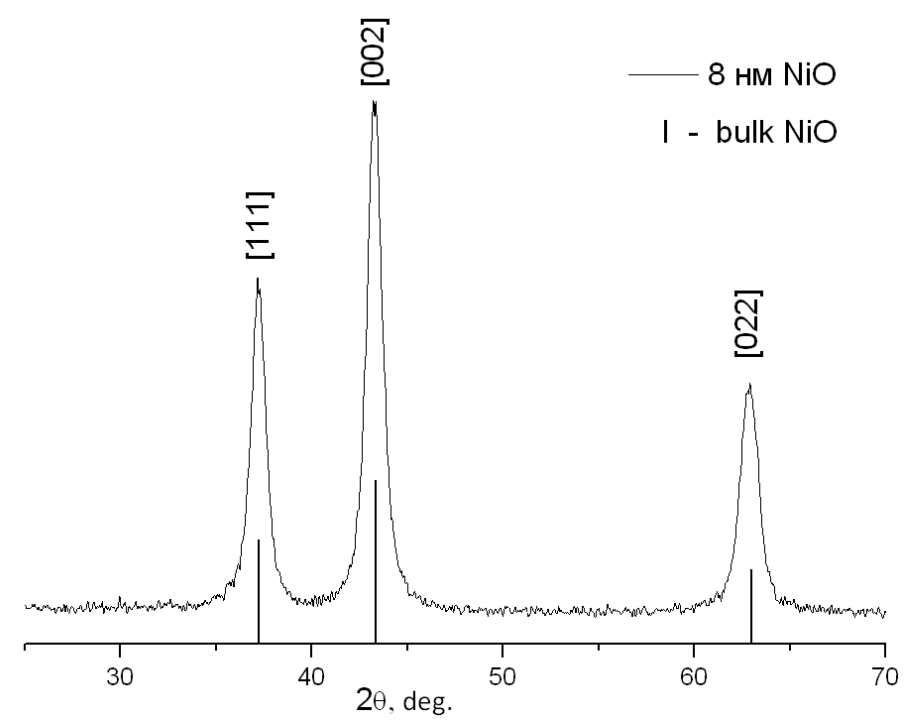

Fig. 1. Experimental XRD pattern of the investigated $\mathrm{NiO}$ nanoparticle sample in comparison with the line diagram illustrating the positions and relative intensities of the bulk $\mathrm{NiO}$ peaks.

\subsection{Measuring the quasi-steady-state magnetic properties}

The magnetic properties under standard quasi-steady-state conditions were measured on a vibrating sample magnetometer (VSM) [19].

Temperature dependences of magnetization $\mathrm{M}(\mathrm{T})$ were measured under zero field cooling (ZFC) and field cooling (FC) conditions. Field dependences of magnetization $\mathrm{M}(\mathrm{H})$ were measured under the ZFC conditions. The field variation rate $\mathrm{dH} / \mathrm{dt}$ during the measurements of the $\mathrm{M}(\mathrm{H})$ hysteresis loops was $60 \mathrm{Oe} / \mathrm{s}$.

\subsection{Measuring the dynamic magnetic hysteresis loops}

The dynamic magnetic hysteresis (DMH) loops were measured on an induction magnetometer in pulsed magnetic fields induced by a standard method of capacitor battery discharge through a solenoid. The possibility of measuring the hysteresis loops is ensured by operation of a setup generating pulsed magnetic fields in the periodic mode. The external field variation rate $\mathrm{dH} / \mathrm{dt}$ depends on two factors: the maximum filed $\mathrm{H}_{\max }$ and pulse length $\tau_{\mathrm{P}}$. The $\tau_{\mathrm{P}}$ value, i.e., the halfperiod time for which the external field changes from $\mathrm{H}=0$ to $\mathrm{H}_{\max }$ and, then, to $\mathrm{H}=0$, was changed by commutation of capacitor battery units to different capacitances. The measurements were performed at $\tau_{\mathrm{P}}$ values of 4,8 , and $16 \mathrm{~ms}$. Figure 2 shows typical time dependences of the solenoid field at different $\mathrm{H}_{\max }$ and $\tau_{\mathrm{P}}$ values. The zero field at the instant of time $\mathrm{t}=\tau_{\mathrm{P}}$ is caused by closing of thyristor units. The magnetic field variation rate at the instant of sample magnetization switching was determined from the $\mathrm{dH} / \mathrm{dt}$ value near a point of $\mathrm{H}=0$ in the experimental $\mathrm{H}(\mathrm{t})$ dependences (Fig. 2). The measurements were performed at a temperature of $77 \mathrm{~K}$. 


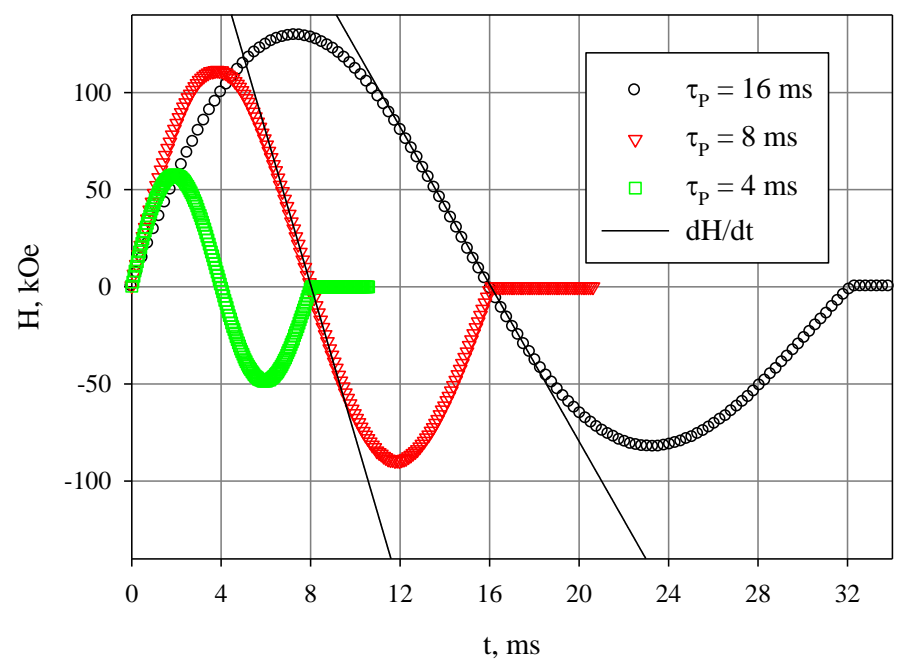

Fig. 2. Time dependences of the solenoid field in the pulsed technique at different maximum applied fields $\mathrm{H}_{\max }$ and specified pulse lengths $\tau_{\mathrm{P}}$. Slopes of the straights correspond to the field variation rate $\mathrm{dH} / \mathrm{dt}$ at the instant of sample magnetization switching.

\section{Results and discussion}

\subsection{SP blocking temperature in the VSM measurements}

Figure 3 shows temperature dependences of magnetization $\mathrm{M}(\mathrm{T})$ for the investigated sample measured under the $\mathrm{ZFC}$ and $\mathrm{FC}(\mathrm{H}=1 \mathrm{kOe})$ conditions. The inset in Fig. 3 presents the data obtained at $\mathrm{H}=100 \mathrm{Oe}$. The shape of these dependences shows that the investigated samples exhibit the characteristic features of the SP behavior, specifically, the pronounced maximum in the $\mathrm{M}(\mathrm{T})_{\mathrm{ZFC}}$ dependence and discrepancy between the $\mathrm{M}(\mathrm{T})_{\mathrm{ZFC}}$ and $\mathrm{M}(\mathrm{T})_{\mathrm{FC}}$ dependences. If we determine the SP blocking temperature $T_{B}$ at the maximum point of the $M(T)_{Z F C}$ dependence, than the $T_{B}$ values are 159 and $164 \mathrm{~K}$ in fields of $\mathrm{H}=1 \mathrm{kOe}$ and $100 \mathrm{Oe}$, respectively; a decrease in the $\mathrm{T}_{\mathrm{B}}$ value with increasing external field is typical of SP systems.

At $\tau_{0} \sim 10^{-11} \mathrm{~s}$ and $\tau_{\mathrm{m}} \sim 10^{1} \mathrm{~s}$ typical of the VSM technique [1], as well as at $\mathrm{d} \approx 8.1 \mathrm{~nm}$ $\left(\mathrm{V} \sim \mathrm{d}^{3}\right)$, the quantity $\mathrm{T}_{\mathrm{B}}=164 \mathrm{~K}$ corresponds, according to Eq. (1), to a magnetic anisotropy constant of about $1.2 \times 10^{6} \mathrm{erg} / \mathrm{cm}^{3}$. This is larger than the magnetic anisotropy constant of bulk $\mathrm{NiO}$ $\left(\mathrm{K}_{\mathrm{V}} \approx 0.8 \times 10^{5} \mathrm{erg} / \mathrm{cm}^{3}\right)$ by an order of magnitude [20]. There are, at least, two factors leading to an increase in the effective magnetic anisotropy constant $\mathrm{K}_{\mathrm{eff}}$ in systems of nanoparticles. The first factor is related to the interparticle interactions [21-23]. The second factor can be the surface anisotropy, which makes the dominant contribution at small particle sizes [24] and is written as $\mathrm{K}_{\mathrm{eff}}=\mathrm{K}_{\mathrm{V}}+6 \mathrm{~K}_{\mathrm{S}} / \mathrm{d}$, where $\mathrm{K}_{\mathrm{S}}$ is the surface magnetic anisotropy constant. 


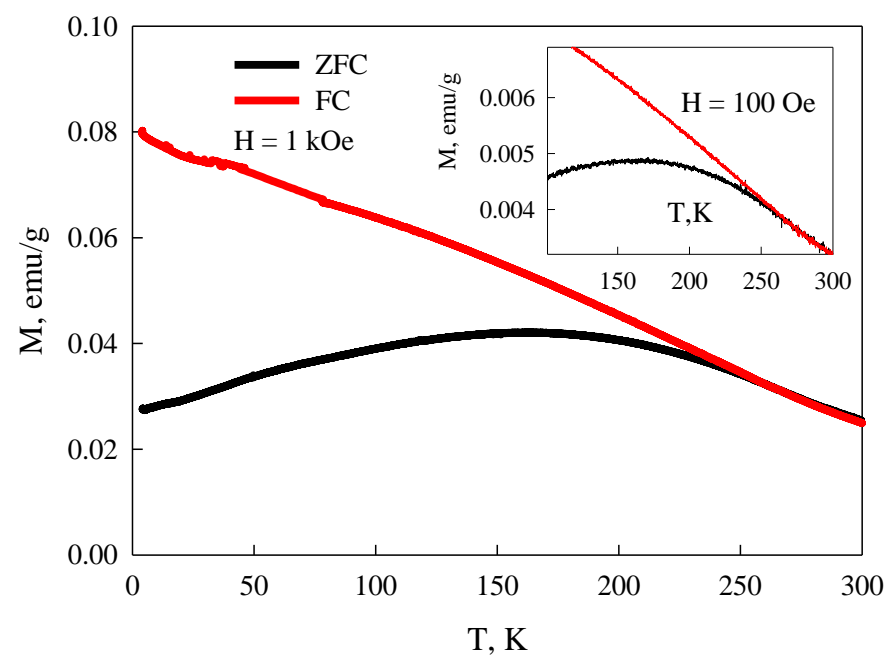

Fig. 3. Temperature dependences of magnetization $\mathrm{M}(\mathrm{T})$ of the investigated $\mathrm{NiO}$ sample $(\mathrm{d} \sim 8 \mathrm{~nm})$ under the ZFC and FC conditions in fields of $1 \mathrm{kOe}$ and 100 Oe (inset).

\subsection{Effect of $H_{\max }$ and $d H / d t$ on the coercivity}

Figure 4 shows selected DMH loops $\left(\tau_{P}=8 \mathrm{~ms}\right.$, the $\mathrm{H}_{\max }$ values are different) together with the data on the magnetic hysteresis loop measured by the VSM technique. One can see no fundamental difference between the shapes of $\mathrm{M}(\mathrm{H})$ dependences obtained using the two techniques. The hysteresis is observed both under the quasi-steady-state conditions $\left(T<\mathrm{T}_{\mathrm{B}}\right)$ and upon dynamic magnetization switching. In strong fields, the functional $\mathrm{M}(\mathrm{H})$ dependence becomes almost linear, which is indicative of the contribution proportional to the field $\chi_{\mathrm{AF}} \times \mathrm{H}$, where $\chi_{\mathrm{AF}}$ is the AFM susceptibility of the particle core $[1,2,5-12,25,26]$.

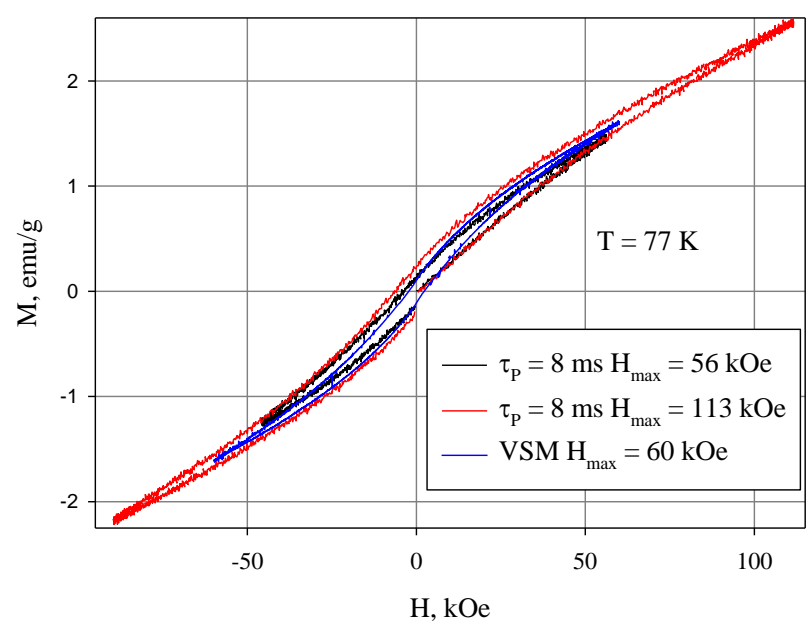

Fig. 4. Magnetic hysteresis loops of the investigated NiO sample $(\mathrm{d} \sim 8 \mathrm{~nm})$ obtained by the pulsed and VSM techniques at the specified pulse lengths $\tau_{\mathrm{P}}$ (for the pulsed technique) and maximum field $\mathrm{H}_{\max }$.

Analysis of the experimental data obtained at different pulse lengths $\tau_{\mathrm{P}}$ and maximum fields $\mathrm{H}_{\max }$ showed that the coercivity is affected by both parameters. Figures 5, 6, and 7 illustrate this behavior. They show enlarged portions of DMH loops near the origin of coordinates. The $\mathrm{H}_{\mathrm{C}}$ values of the DMH loops were determined as crossing points of the $\mathrm{M}(\mathrm{H})$ dependences and abscissa axis $(\mathrm{M}=0)$ in the negative field region. 


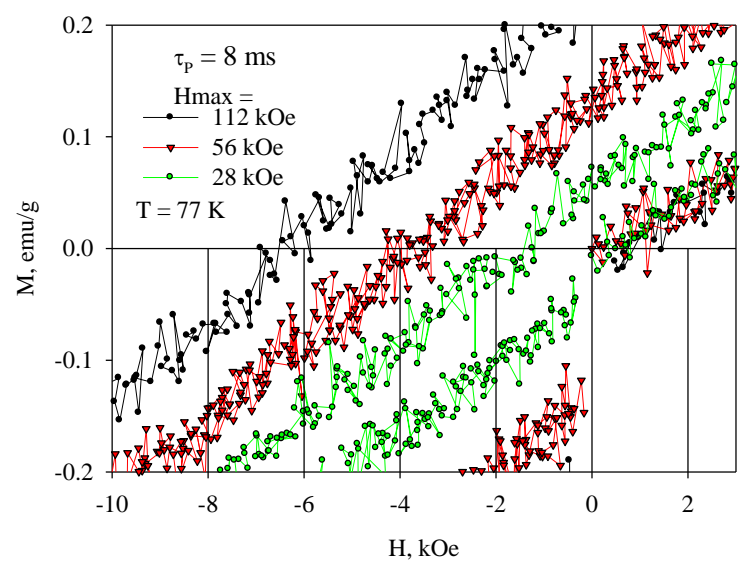

Fig. 5. Portions of the $\mathrm{M}(\mathrm{H})$ hysteresis loops of the investigated $\mathrm{NiO}$ sample $(\mathrm{d} \sim 8 \mathrm{~nm})$ near the origin of coordinates upon pulsed magnetization switching in different maximum applied field $\mathrm{H}_{\max }$. The pulse length is constant $\left(\tau_{\mathrm{P}}=8 \mathrm{~ms}\right) . \mathrm{T}=77 \mathrm{~K}$.

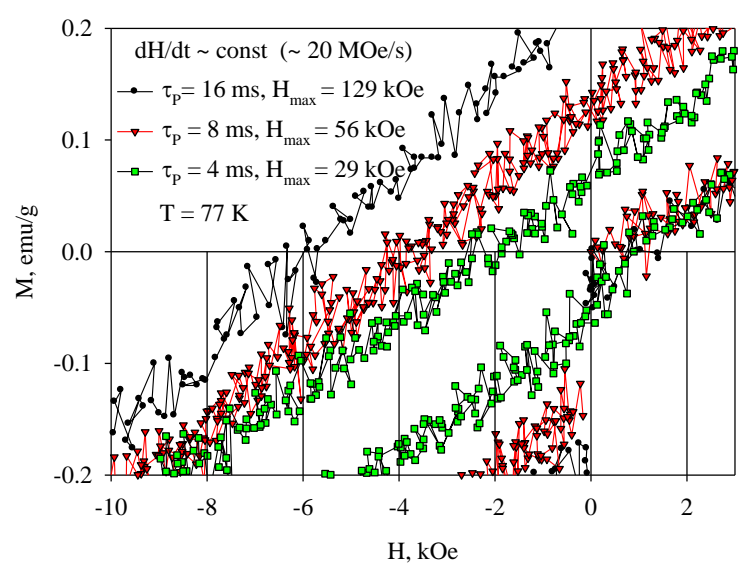

Fig. 6. Portions of the $\mathrm{M}(\mathrm{H})$ hysteresis loops of the investigated $\mathrm{NiO}$ sample $(\mathrm{d} \sim 8 \mathrm{~nm})$ near the origin of coordinates upon pulsed magnetization switching at approximately the same field variation rate of $\mathrm{dH} / \mathrm{dt} \approx 20 \mathrm{MOe} / \mathrm{s}$. The pulse lengths $\tau_{\mathrm{P}}$ and maximum applied fields $\mathrm{H}_{\mathrm{max}}$ are different (presented in the figure). $\mathrm{T}=77 \mathrm{~K}$. 


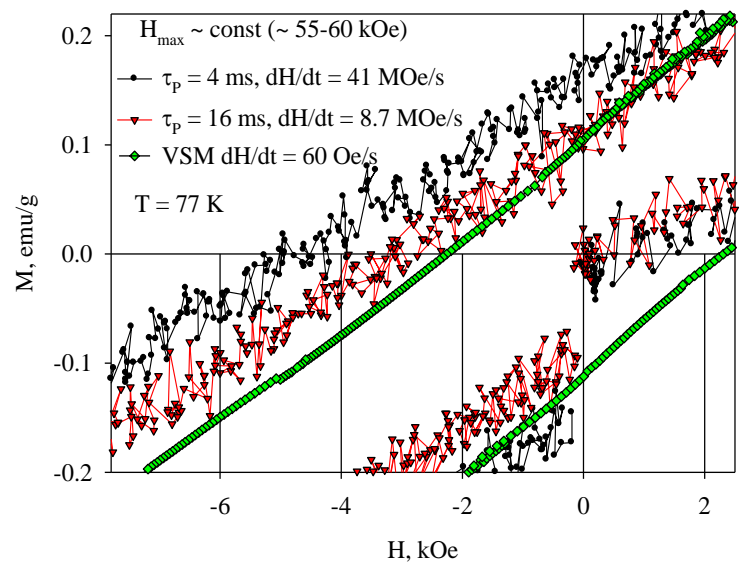

Fig. 7. Portions of the $\mathrm{M}(\mathrm{H})$ hysteresis loops of the investigated $\mathrm{NiO}$ sample $(\mathrm{d} \sim 8 \mathrm{~nm})$ near the origin of coordinates for pulsed magnetization switching and VSM. The maximum applied field $\mathrm{H}_{\max }$ is approximately the same in all the three dimensions. The pulse lengths $\tau_{\mathrm{P}}$ and field variation rates $\mathrm{dH} / \mathrm{dt}$ are presented in the figure.

Figure 5 shows the selected data obtained at $\tau_{\mathrm{P}}=8 \mathrm{~ms}$ and different maximum fields $\mathrm{H}_{\max }$. It can be seen that with increasing $\mathrm{H}_{\max }$ the coercivity grows. The increase in $\mathrm{H}_{\max }$ at $\tau_{\mathrm{P}}=$ const leads to an increase in the field variation rate $\mathrm{dH} / \mathrm{dt}$. However, at the constant parameter $\mathrm{dH} / \mathrm{dt}$ the $\mathrm{H}_{\mathrm{C}}$ value increases with the maximum applied field. This is illustrated in Fig. 6, which shows the selected data obtained at the approximately equal values $\mathrm{dH} / \mathrm{dt} \approx 20 \mathrm{MOe} / \mathrm{s}$. When the $\mathrm{H}_{\max }$ value is fixed and the field variation rate is changed by changing the pulse length, then the increase in $\mathrm{dH} / \mathrm{dt}$ also leads to the coercivity growth. This behavior is illustrated in Fig. 7, where the pulsed magnetization switching data are presented together with the $\mathrm{M}(\mathrm{H})$ hysteresis loop obtained under the quasi-steady-state conditions (VSM, dH/dt $=60 \mathrm{Oe} / \mathrm{s})$.

Figure 8 shows consolidated data on the effect of field variation rate (Fig. 8a) and maximum applied field (Fig. 8b) on the $\mathrm{H}_{\mathrm{C}}$ value. The data are grouped in accordance with the pulse length. One can clearly see that the coercivity increases with $\mathrm{H}_{\max }$ and $\mathrm{dH} / \mathrm{dt}$. 

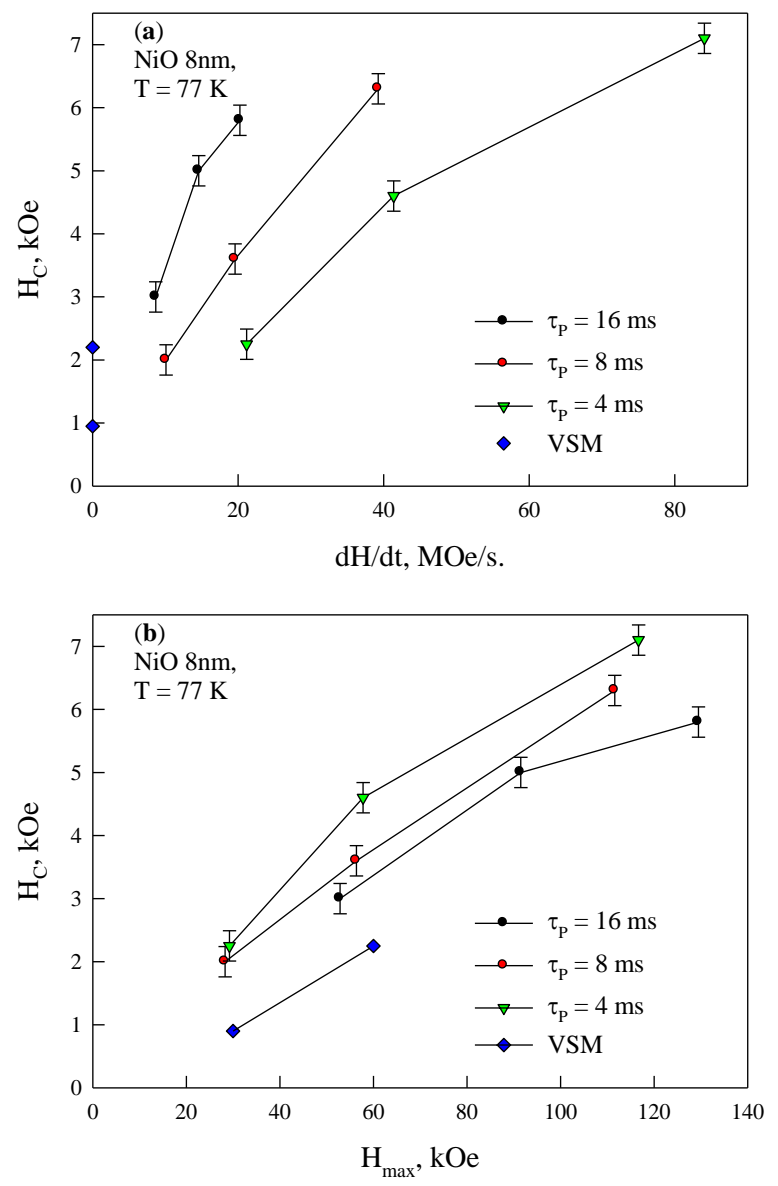

Fig. 8. Dependences of the coercivity $\mathrm{H}_{\mathrm{C}}$ on (a) the field variation rate $\mathrm{dH} / \mathrm{dt}$ and (b) maximum applied field $\mathrm{H}_{\max }$ upon pulsed magnetization switching for the investigated $\mathrm{NiO}$ sample $(\mathrm{d} \sim 8 \mathrm{~nm})$ at $\mathrm{T}=77 \mathrm{~K}$. The data grouped in accordance with pulse lengths $\tau_{\mathrm{P}}$ are shown by different symbols. For comparison, the data of the quasi-steady-state magnetic measurements (VSM) are presented.

In our previous study on dynamic magnetization switching of $\varepsilon-\mathrm{Fe}_{2} \mathrm{O}_{3}$ nanoparticles [16], we established that the main parameter determining the coericivity is the field variation rate $\mathrm{dH} / \mathrm{dt}$. For the $\varepsilon-\mathrm{Fe}_{2} \mathrm{O}_{3}$ nanoparticles, the field at which the $\mathrm{M}(\mathrm{H})$ dependence becomes reversible is about 50 $60 \mathrm{kOe}$. In AFM NiO, along with the parameter $\mathrm{dH} / \mathrm{dt}$, an additional factor, specifically, the maximum applied field, arises. At the same time, the coercivity increases with a decrease in the pulse length (an increase in $\mathrm{dH} / \mathrm{dt}$ ) at $\mathrm{H}_{\max } \approx$ const. This can be seen in Figs. 7 and Fig. $8 \mathrm{~b}$ (see the "cross section" of the $\mathrm{H}_{\mathrm{C}}\left(\mathrm{H}_{\max }\right)$ dependence at $\mathrm{H}_{\max }$ values in the vicinity of $\left.60 \mathrm{kOe}\right)$. This trend is typical of the relaxation processes investigated upon dynamic magnetization switching in magnetic particles [13-15]. At $\mathrm{H}_{\max }=$ const, a decrease in the pulse length is equivalent to an increase in the ac field frequency. We can estimate the increment of the SP blocking temperature in our experiments. Assuming $\tau_{\mathrm{m}}$ in Eq. (1) to be the hysteresis loop variation time, we obtain $\tau_{\mathrm{m}} \sim 10^{4} \mathrm{~s}$ for the VSM measurements. In the case of ac fields with frequency $\omega$, we obviously have $\tau_{\mathrm{m}}=2 \pi / \omega=2 \tau_{\mathrm{P}}$. Then, at the minimum pulse length $\tau_{\mathrm{P}}=4 \mathrm{~ms}$, we obtain (at $\tau_{0} \sim 10^{-10}-10^{-11} \mathrm{~s}$ ) that the $\mathrm{T}_{\mathrm{B}}$ value increases by a factor of about 1.7 (to $\sim 280 \mathrm{~K}$ ) relative to the case of the quasi-steady-state conditions. The broadening of the temperature range corresponding to the blocked state (hysteresis) is equivalent to an increase in $\mathrm{H}_{\mathrm{C}}$ at $\mathrm{T}=\mathrm{const}\left(\mathrm{T}<\mathrm{T}_{\mathrm{B}}\right)$. The coercivity growth with increasing ac frequency $\left(\omega=\pi / \tau_{\mathrm{P}}\right)$ does not contradict the theoretical results obtained for AFM nanoparticles in [15]; however, at the current stage of investigations, it is hard to make a comparison with the theory. 
Let us get back to the effect of $\mathrm{H}_{\max }$ on the coercivity. In our opinion, the observed effect is caused by the fact that the $\mathrm{H}_{\max }$ value is lower than the field at which the $\mathrm{M}(\mathrm{H})$ dependence becomes reversible. In the quasi-steady-state magnetic measurements at $\mathrm{T}=77 \mathrm{~K}$ and an external field of $60 \mathrm{kOe}$, the $\mathrm{M}(\mathrm{H})$ hysteresis loop is still open. Such a behavior of the AFM nanoparticles was established fairly long ago (see, for example, [27]). In [28], it was experimentally demonstrated and justified that the $\mathrm{H}_{\mathrm{C}}\left(\mathrm{H}_{\max }\right)$ dependence for AFM nanoparticles is $S$-shaped. In addition, the saturation fields of the low-temperature $\mathrm{H}_{\mathrm{C}}\left(\mathrm{H}_{\max }\right)$ dependence of the AFM ferrihydrite studied in [28] are hundreds of kOe at low temperatures. In [29], we used a similar approach to clarify the origin of shift of the magnetic hysteresis loop of ferrihydrite nanoparticles after cooling in an external field (if the loop at a certain $\mathrm{H}_{\max }$ value remains open, then the loop shift observed under the FC conditions at $\mathrm{H}_{\text {cool }}=\mathrm{H}_{\max }$ can reflect the minor loop effect). We may conclude that the AFM nanoparticles have fairly high fields of irreversible behavior of the $\mathrm{M}(\mathrm{H})$ dependence. Possibly, at pulsed (ac) magnetic fields, this AFM nanoparticle irreversibility field additionally increases over the field for the quasisteady-state conditions. It can be seen in Fig. 8b that the $\mathrm{H}_{\mathrm{C}}\left(\mathrm{H}_{\max }\right)$ dependences are far from saturation $^{1}$. Therefore, the maximum applied field is a factor that mainly determines the $\mathrm{DMH}$ behavior of AFM nanoparticles.

\section{Conclusions}

The dynamic magnetization switching of AFM nickel oxide particles $8 \mathrm{~nm}$ in size was experimentally investigated. Using the pulsed field technique, the coercivity growth upon pulsed magnetization switching relative to the quasi-steady-state magnetic hysteresis measurements was found. It was shown that, similar to single-domain FM nanoparticles, the coercivity of the investigated nickel oxide sample increases with a decrease in the pulse length (with an increase in frequency), which is related to the relaxation processes.

However, the other factor affecting significantly the coercivity is the maximum applied field. Admittedly, in the investigated sample at $\mathrm{T}=77 \mathrm{~K}\left(\mathrm{~T}_{\mathrm{B}}=164 \mathrm{~K}\right.$ in the quasi-steady-state magnetic measurements), the DMH loops remain open up to $\mathrm{H}_{\max } \sim 130 \mathrm{kOe}$. As a result, the coercivity depends on this parameter as well (increases with $\mathrm{H}_{\max }$ at all other conditions being equal). We believe that this feature is typical of not only the investigated sample, but also of a whole class of nanoparticles with the AFM ordering and uncompensated magnetic moment (according to our data, this conclusion is valid also for AFM ferrihydrite nanoparticles). This should be taken into account when building the theory of dynamic magnetic hysteresis of AFM nanoparticles. Note that the significant coercivity growth and, consequently, the increase in the hysteresis loop square and heat loss in the pulsed technique as compared with the quasi-steady-state magnetic measurements can broaden the field of application of AFM nanoparticle-based materials.

We are grateful to Yu.V. Knyazev, S.V. Semenov, and M.I. Kolkov for help.

This study was supported by the Russian Foundation for Basic Research, Government of the Krasnoyarsk Territory, and the Krasnoyarsk Territorial Foundation for Support of Scientific and R\&D Activities, project no. 17-42-240138.

\section{References}

${ }^{1}$ Note that, according to the results of quasi-steady-state magnetic measurements of the $\mathrm{NiO}$ sample at $\mathrm{T}=4.2 \mathrm{~K}$ in fields of up to $\mathrm{H}_{\max }=75 \mathrm{kOe}$, the $\mathrm{H}_{\mathrm{C}}\left(\mathrm{H}_{\max }\right)$ dependence has a positive curvature; i.e., in fields of up to $75 \mathrm{kOe}$, there is no trend to saturation, which confirms the authors' assumption. 
1. S. Mørup, D.E. Madsen, C. Fradsen, C.R.H. Bahl, and M.F. Hansen, J. Phys.: Condens. Matter 19, 213202 (2007).

2. Yu.L. Raikher and V.I. Stepanov, J. Phys.: Condens. Matter. 20, 204120 (2008).

3. Q.A. Pankhurst, N.T.K. Thanh, S.K. Jones, J. Dobson, J. Phys. D 42, 224001 (2009).

4. K. Dobretsov, S. Stolyar, A. Lopatin. Acta Otorhinolaryngol. Ital. 35, 2, 97 (2015)

5. S.A. Makhlouf, F.T. Parker, F.E. Spada, and A.E. Berkowitz, J. Appl. Phys. 81(8), 5561 (1997).

6. S.A. Makhlouf, F.T. Parker, A.E. Berkowitz, Phys. Rev. B 55, R14717 (1997).

7. S.A. Makhlouf, H. Al-Attar, and R.H. Kodama, Solid State Commun. 145, 1 (2008).

8. M.S. Seehra, A. Punnoose, Solid State Communications 128299 (2003).

9. N.J.O. Silva, V.S. Amaral, and L.D. Carlos, Phys. Rev. B 71, 184408 (2005).

10. D.A. Balaev, A.A. Dubrovskiy, A.A. Krasikov, S.I. Popkov, A.D. Balaev, K.A. Shaikhutdinov, V.L. Kirillov, and O. N. Mart'yanov, Physics of the Solid State, 59, (N8), 1547 (2017).

11. Chandni Rani, S.D. Tiwari, J. Magn. Magn. Mater. 385, 272-276 (2015).

12. D.A. Balaev, S.I. Popkov, A.A. Krasikov, A.D. Balaev, A.A. Dubrovskiy, S.V. Stolyar, R.N. Yaroslavtsev,V.P. Ladygina, and R.S. Iskhakov, Physics of the Solid State, 59, (N 10), 1940 (2017).

13. I. S. Poperechny, Yu. L. Raikher, and V. I. Stepanov, Phys. Rev. B 82, 174423 (2010).

14. I. S. Poperechny and Yu. L. Raikher, Physica B 435, 58-61 (2014).

15. Yu.P. Kalmykov, B. Ouari, and S.V. Titov, J. Appl. Phys. 120, 053901 (2016).

16. D.A. Balaev, I.S. Poperechny, A.A. Krasikov, K.A. Shaikhutdinov, A.A. Dubrovskiy, S.I. Popkov, A.D. Balaev, S.S. Yakushkin, G.A. Bukhtiyarova, O.N. Martyanov, and Yu.L. Raikher, J. Appl. Phys. 117, 063908 (2015).

17. S. S. Yakushkin, A. A. Dubrovskiy, D. A. Balaev, K. A. Shaykhutdinov, G. A. Bukhtiyarova et al. J. Appl. Phys. 111, 044312 (2012).

18. D. A. Balaev, A. A. Dubrovskiy, K. A. Shaykhutdinov, O. A. Bayukov, S. S. Yakushkin, G. A. Bukhtiyarova, and O. N. Martyanov, J. Appl. Phys. 114, 163911 (2013).

19. A.D. Balaev, Yu.V. Boyarshinov, M.M. Karpenko, and B.P. Khrustalev, Prib. Tekh. Eksp., 3, 167 (1985).

20. M. Tadic, D. Nikolic, M. Panjan, and G. R. Blake, J.Alloys Compd. 647, 1061 (2015).

21. H. Shim, P. Dutta, M.S. Seehra, J. Bonevich, Solid State Communications 145 192, (2008).

22. T.S. Berquó, J.J. Erbs, A. Lindquist, R.L. Penn, and S.K. Banerjee, J. Phys.: Condens. Matter 21, 176005 (2009).

23. D.A. Balaev, S.V. Semenov, A.A. Dubrovskiy, S.S. Yakushkin, V.L. Kirillov, O.N. Martyanov, J. Magn. Magn. Mater. 440199 (2017).

24. F. Bødker, S. Mørup, and S. Linderoth, Phys. Rev. Lett. 72, 282 (1994).

25. D.A. Balaev, A.A. Krasikov, A.A. Dubrovskiy, S.I. Popkov, S.V. Stolyar, O.A. Bayukov, R.S. Iskhakov, V.P. Ladygina, R.N. Yaroslavtsev, J. Magn. Magn. Mater. 410, 171 (2016).

26. Ch. Rani, S.D. Tiwari, Physica B 513, 58 (2017).

27. R.H. Kodama, A.E. Berkowitz, Phys. Rev. B 59, 6321 (1999).

28. N. J. O. Silva, V. S. Amaral, A. Urtizberea, R. Bustamante, A. Milláan, F. Palacio, E. Kampert, U. Zeitler, S. de Brion, O. Iglesias, and A. Labarta, Phys. Rev. B 84, 104427 (2011). 
29. D.A. Balaev, A.A. Krasikov, A.A. Dubrovskiy, S.I. Popkov, S.V. Stolyar, R.S. Iskhakov, V.P. Ladygina, and R.N. Yaroslavtsev, J. Appl. Phys. 120, 183903 (2016).

\section{Figure captions}

Fig. 1. Experimental XRD pattern of the investigated $\mathrm{NiO}$ nanoparticle sample in comparison with the line diagram illustrating the positions and relative intensities of the bulk $\mathrm{NiO}$ peaks.

Fig. 2. Time dependences of the solenoid field in the pulsed technique at different maximum applied fields $\mathrm{H}_{\max }$ and specified pulse lengths $\tau_{\mathrm{P}}$. Slopes of the straights correspond to the field variation rate $\mathrm{dH} / \mathrm{dt}$ at the instant of sample magnetization switching.

Fig. 3. Temperature dependences of magnetization $\mathrm{M}(\mathrm{T})$ of the investigated $\mathrm{NiO}$ sample $(\mathrm{d} \sim 8 \mathrm{~nm})$ under the ZFC and FC conditions in fields of $1 \mathrm{kOe}$ and 100 Oe (inset).

Fig. 4. Magnetic hysteresis loops of the investigated $\mathrm{NiO}$ sample $(\mathrm{d} \sim 8 \mathrm{~nm})$ obtained by the pulsed and VSM techniques at the specified pulse lengths $\tau_{\mathrm{P}}$ (for the pulsed technique) and maximum field $\mathrm{H}_{\max }$.

Fig. 5. Portions of the $\mathrm{M}(\mathrm{H})$ hysteresis loops of the investigated $\mathrm{NiO}$ sample $(\mathrm{d} \sim 8 \mathrm{~nm})$ near the origin of coordinates upon pulsed magnetization switching in different maximum applied field $\mathrm{H}_{\max }$. The pulse length is constant $\left(\tau_{\mathrm{P}}=8 \mathrm{~ms}\right) . \mathrm{T}=77 \mathrm{~K}$.

Fig. 6. Portions of the $\mathrm{M}(\mathrm{H})$ hysteresis loops of the investigated $\mathrm{NiO}$ sample $(\mathrm{d} \sim 8 \mathrm{~nm})$ near the origin of coordinates upon pulsed magnetization switching at approximately the same field variation rate of $\mathrm{dH} / \mathrm{dt} \approx 20 \mathrm{MOe} / \mathrm{s}$. The pulse lengths $\tau_{\mathrm{P}}$ and maximum applied fields $\mathrm{H}_{\mathrm{max}}$ are different (presented in the figure). $\mathrm{T}=77 \mathrm{~K}$.

Fig. 7. Portions of the $\mathrm{M}(\mathrm{H})$ hysteresis loops of the investigated $\mathrm{NiO}$ sample $(\mathrm{d} \sim 8 \mathrm{~nm})$ near the origin of coordinates for pulsed magnetization switching and VSM. The maximum applied field $\mathrm{H}_{\max }$ is approximately the same in all the three dimensions. The pulse lengths $\tau_{\mathrm{P}}$ and field variation rates $\mathrm{dH} / \mathrm{dt}$ are presented in the figure.

Fig. 8. Dependences of the coercivity $\mathrm{H}_{\mathrm{C}}$ on (a) the field variation rate $\mathrm{dH} / \mathrm{dt}$ and (b) maximum applied field $\mathrm{H}_{\max }$ upon pulsed magnetization switching for the investigated NiO sample $(\mathrm{d} \sim 8 \mathrm{~nm})$ at $\mathrm{T}=77 \mathrm{~K}$. The data grouped in accordance with pulse lengths $\tau_{\mathrm{P}}$ are shown by different symbols. For comparison, the data of the quasi-steady-state magnetic measurements (VSM) are presented. 